\title{
The Temperature Dependent Anisotropy Influence on the Magnetic Behavior of Thin Films
}

\author{
E. BÎRSAN* \\ Department of Physics, "Lucian Blaga" University, Dr. I. Ratiu Str., No. 5-7, Sibiu, 550012, Romania \\ AND R. CHIŞ \\ Faculty of Engineering "Hermann Oberth", "Lucian Blaga" University, Sibiu, Romania
}

(Received April 4, 2011)

\begin{abstract}
The magnetism of the layered materials attracts a great interest in the last years. In this context, it is necessary to take into account the anisotropic comportment of this kind of systems, due to its important influence on the magnetic properties that characterize these structures. In this paper, we intend to study in a numerical manner the effect of the temperature dependence of the anisotropic parameters on the specific behavior of a two-dimensional square lattice of unitary magnetic spins. Thus, using the Monte Carlo technique, we calculate the magnetic susceptibility and the specific heat of the considered physical system governed by a Heisenberg type Hamiltonian and we determine the magnetic properties of the system, induced by the temperature dependence of anisotropy that characterizes the thin film. In this context, we point out a slow decrease of the critical temperature specific to the ferromagnetism-paramagnetism phase transition, in comparison with the case of constant anisotropy. We also detect a critical temperature slight decrease along with the increasing slope of the anisotropy linear variation as a function of temperature.
\end{abstract}

PACS: $75.70 . \mathrm{Ak}, 75.30 . \mathrm{Gw}, 75.40 . \mathrm{Mg}$

\section{Introduction}

In the current physics, the magnetism of thin films and generally of nanostructured materials represents a field of great interest [1]. In this context, the study of the multilayer magnetic structures holds a special place. The theoretical and experimental approaches dedicated to multilayered magnetic thin films revealed the distinctive characteristics of these structures $[2,3]$. It is also important to note that in the last years, the numerical methods were intensively used for the study of the magnetic properties of the thin films, these approaches having the versatility of the used physical model as an advantage, due to the possibility of choosing the physical parameters in various ways. Therefore, the special characteristics of these structures can be determined, by emphasizing the role of some interactions which can occur in the system and voluntarily neglecting other factors of influence. In this context, the structure of the studied systems plays an important role. As follows, in the case of the thin films it is strictly necessary to take into account the anisotropic factors, because they have a major influence on the magnetic properties of these systems $[4,5]$. Another extremely important factor in this con-

\footnotetext{
* corresponding author; e-mail: birsan.eugen@gmail.com
}

text is the temperature dependence of anisotropy. Thus, the study of the temperature dependence of anisotropic parameters and their influence on the magnetic properties of this kind of physical systems can be very interesting [6-9], regarding the specific phenomena that can appear. Consequently, in this paper we intend to study, using a numerical approach, the influence of the temperature variation of anisotropy on the magnetic behavior of two-dimensional spins lattice that can represent a good approximation for a thin film.

The paper is organized as follows: in Sect. 2 we present the physical model and the numerical algorithm used in our numerical simulation, Sect. 3 is dedicated to the numerical results and the final conclusions are presented in Sect. 4.

\section{The physical model and the numerical algorithm}

We consider a two-dimensional square lattice of unitary magnetic spins $(S=1)$, with a lattice constant $a=1$, characterized by open boundary conditions (OBC) for the directions $O x$ and $O y$. The magnetic spins can arbitrarily move around a fix point and the interactions between the lattice spins are governed by the next Hamiltonian [10]: 


$$
H=-\sum_{\langle i, j\rangle} J_{i j} \boldsymbol{S}_{i} \cdot \boldsymbol{S}_{j}-\sum_{i} K_{i} S_{i z}^{2}+\sum_{i, j} D_{i j} T_{i j} .
$$

The first term of the Hamiltonian (1) means the ferromagnetic direct exchange interaction between the adjacent magnetic spins, the second term represents the crystalline anisotropy interaction (favors the out-of-plane orientation of the spins) and the last term denotes the long range dipolar interaction (favors the in-plane alignment of the magnetic spins). The dipolar interaction is characterized by the expression

$$
T_{i j}=\frac{\boldsymbol{S}_{i} \cdot \boldsymbol{S}_{j}}{r_{i j}^{3}}-3 \frac{\left(\boldsymbol{S}_{i} \cdot \boldsymbol{r}_{i j}\right)\left(\boldsymbol{S}_{j} \cdot \boldsymbol{r}_{i j}\right)}{r_{i j}^{5}},
$$

where $r_{i j}$ is the distance between the magnetic spins involved in the interaction and $\boldsymbol{S}_{i}$ represents the spin vector corresponding to the position " $i$ " of the lattice.

In our model we assume that the crystalline anisotropy follows the linear dependence with temperature, given by the expression

$$
K=K_{0}(\alpha+\beta T) .
$$

The aim of this paper is mainly to emphasize the influence of the linear temperature dependence of the crystalline anisotropy on the magnetic behavior of the thin film. In our numerical study, we apply the Monte Carlo technique $[11,12]$, using the standard Metropolis algorithm [13]. Thus, we run $10^{3}$ Monte Carlo steps per spin for thermalization of the system and $10^{3}$ Monte Carlo steps per spin to calculate the physical quantities of interest. In the following, we present the physical quantities we have in view [14], in order to perform the specified purpose of the paper - the out-of-plane magnetizations per spin are given by the expression

$$
\tilde{m}_{z}=\frac{\sum_{i} S_{i z}}{N}
$$

In the next, we use the relative magnetization per spin (normalized to unity)

$$
m_{z}=\frac{\tilde{m}_{z}}{\tilde{m}_{z \text { sat }}},
$$

$\tilde{m}_{z \text { sat }}$ being the saturation magnetization per spin. Another extremely important quantity we consider in our work is the out-of-plane magnetic susceptibility, calculated using the expression

$$
\chi_{z}=N \frac{\left\langle\tilde{m}_{z}^{2}\right\rangle-\left\langle\tilde{m}_{z}\right\rangle^{2}}{k_{\mathrm{B}} T},
$$

where $N$ is the number of the lattice spins, $k_{\mathrm{B}}$ is the Boltzmann constant (considered unitary) and $T$ is the absolute temperature of the system. We also note that \langle\rangle means the statistical average over the accepted states obtained in the numerical simulation. At the same time, we introduce the relation of the specific heat

$$
c=N \frac{\left\langle E^{2}\right\rangle-\langle E\rangle^{2}}{k_{\mathrm{B}} T^{2}},
$$

where $E$ is the energy per spin. In the next, the energy is measured in $J_{0} S^{2}$ and the unit for temperature is $\frac{J_{0} S^{2}}{k_{\mathrm{B}} T}$ ( $J_{0}=1$ being the direct exchange reference parameter).

\section{The simulations results}

In this section, we present the results obtained in our numerical simulations, for different settings of the parameters values that characterized the studied system, corresponding to the physical model we assumed in the previous section. It is important to note here that our study is focused on the area of the phase diagram characterized by a weak dipolar interaction, which favors a magnetization perpendicular to the film plane at low temperatures (this magnetic ordering cancel with temperature increase and the system evolves to paramagnetic phase).

\subsection{The case of constant anisotropy}

Firstly, we investigate the magnetic behavior of the above presented system with the dimensions $N_{x}=N_{y}=$ $50, N_{z}=1$, if we assume that the anisotropy does not depend on temperature. The physical system is characterized by the following parameter values: $K_{0}=1.00$, $\alpha=1, \beta=0, D=0.115$, for three different direct exchange parameters values $J=1.00,1.10,1.20$. Thus, if the anisotropy parameter variation with temperature is absent, we study the out-of-plane magnetic susceptibility temperature dependence (Fig. 1a). The peaks of the graph suggest that in the system there appears a magnetic phase transition, along with the temperature increase [15]. The network spins go from ferro-

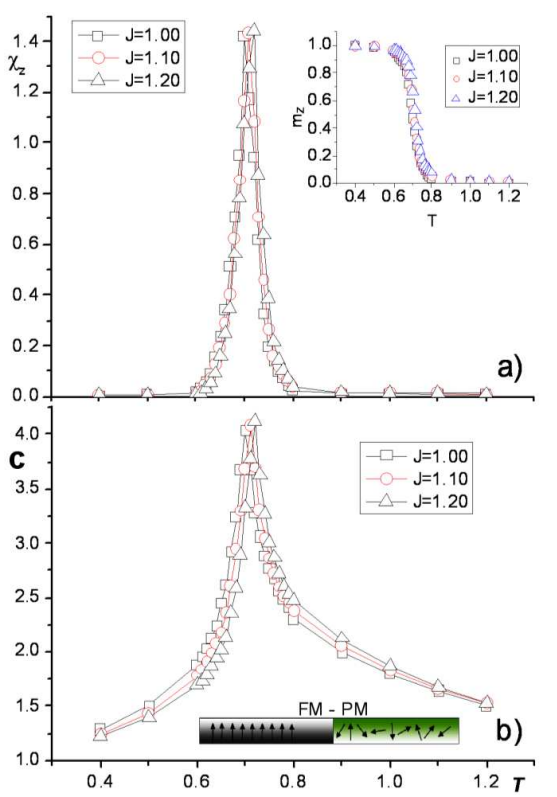

Fig. 1. The out-of-plane magnetic susceptibility (a) and the specific heat (b) vs. temperature for $K_{0}=1.00$, $\alpha=1, \beta=0, D=0.115$ and three different direct exchange interaction parameter values. The insets show the out-of-plane magnetization per spin (a) and the lattice spin structure vs. temperature (b). 
magnetic ordering to the disordered phase and in this context we observe the critical temperature increasing with the direct exchange interaction parameter. The out-of-plane magnetization form, presented in the inset of Fig. 1a attests the ferromagnetism-paramagnetism (FM-PM) phase transition described above. The specific heat variation as a function of temperature presented in Fig. 1b confirms the existence of the above mentioned phase transition and the influence of the direct exchange parameter increase on the magnetic stability of the system (the structures of the two magnetic phases are shown in the inset). In other words, we can observe the rise of the magnetic ordered phase stability with the direct exchange interaction parameter increase. Therefore, in this context, we deal with two competitive processes: firstly we note the tendency of magnetic ordering, favored by the direct exchange interaction between the magnetic spins of the lattice and by the long range dipolar interaction that induces the magnetic domains forming, and on the other hand, we have in view the thermal disorder of the physical system, that implies the magnetic ordered phase stability weakening.

\subsection{The case of uniform temperature dependence of anisotropy}

In this section, we intend to determine the direct exchange variation influence on the magnetic properties of the system, for a single decreasing slope of anisotropy as a function of temperature. Thus, for a lattice with the dimensions $N_{x}=N_{y}=50, N_{z}=1$, we assume the next parameters values $K_{0}=1.00, \alpha=1, \beta=-0.05, D=0.115$, for the three different direct exchange parameters values $J=1.00,1.10,1.20$. In this case, taking into account the temperature dependence of the anisotropy parameter (characteristic for the $\mathrm{Co} / \mathrm{Au}$ thin films [16]) the out-of-plane magnetic susceptibility, shown in Fig. 2a, reveals a slow decrease of the critical temperature in comparison with the case of constant anisotropy. The inset of Fig. 2a presents the percentage variation of the critical temperature, calculated with the relation

$$
\Delta T / T=\frac{T_{\mathrm{cr} 0}-T_{\mathrm{cr}}}{T_{\mathrm{cr} 0}},
$$

where $T_{\text {cr }}$ is the critical temperature in the case treated in Sect. 3.2, when the anisotropy parameter is dependent on temperature and $T_{\text {cro }}$ is the critical temperature in the case presented in Sect. 3.1, where the anisotropy is constant in all the studied temperature interval, for the three different direct exchange interaction parameters. The relative variation of the critical temperature presents a linear drop, along with the direct exchange parameter increasing. The influence of the temperature variation of anisotropy parameter, on the magnetic ordering of the system is more acute at low direct exchange interaction values. This influence decreases with the direct exchange interaction parameter enhancement. At the same time, the direct exchange interaction increase leads to the magnetic ordered phase stability improvement. We also ob- serve that the critical temperature variation with the direct exchange interaction parameter, obtained from the study of the specific heat (Fig. 2b) is in good agreement with the above described behavior of the system.

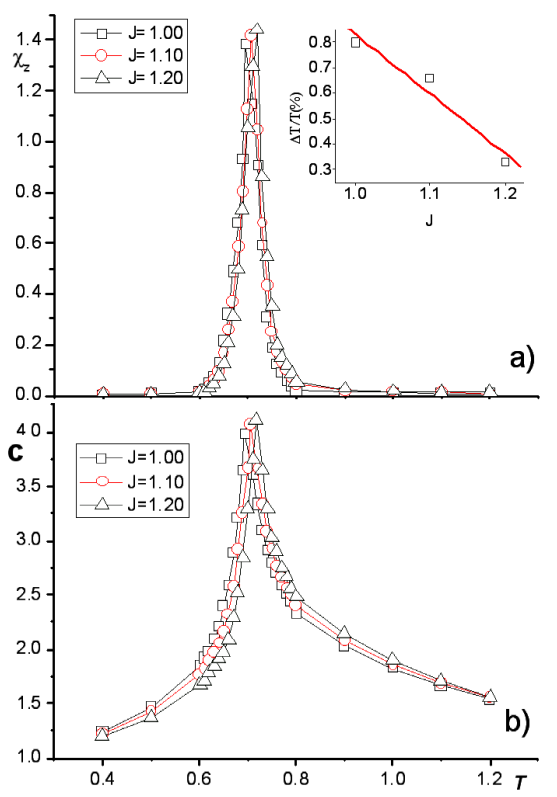

Fig. 2. The out-of-plane magnetic susceptibility (a) and the specific heat (b) vs. temperature for $K_{0}=1.00$, $\alpha=1, \beta=-0.05, D=0.115$ and three different direct exchange interaction parameter values. In inset we show the relative variation of the critical temperature vs. direct exchange parameter.

\subsection{The finite size study}

In the current section we study the finite size effect for three dimensions of the lattice $\left(N_{x}=N_{y}=50,60,70\right.$; $N_{z}=1$ ) and the following physical parameters involved in the numerical simulation: $J=1.00, K_{0}=1.00, \alpha=1$, $\beta=-0.05, D=0.115$. In this context, we investigate the system behavior regarding the above mentioned magnetic phase transition. Figure 3 shows the out-of-plane magnetic susceptibility in these conditions, for three different lattice dimensions. One observes a slow increase of the critical temperature with the lattice dimension. The relative variation of the critical temperature (presented in the inset of Fig. 3) is given by the relation

$$
\Delta T / T=\frac{T_{\mathrm{cr}, N}-T_{\mathrm{cr}, 50}}{T_{\mathrm{cr}, 50}}, \quad N=60,70,
$$

where $T_{\mathrm{crN}}$ is the critical temperature that characterized the lattice with the dimension $N$ (the reference size of the lattice is $N=50$ ). The slope that characterizes the linear approximation of the critical temperature relative variation is of $9.7 \%$ in this range of the lattice dimensions. In the other words, assuming the physical conditions presented above, for a lattice dimension enlargement of $16.6 \%$ (from 60 to 70 ), we observe a critical temperature increase of $0.95 \%$. 


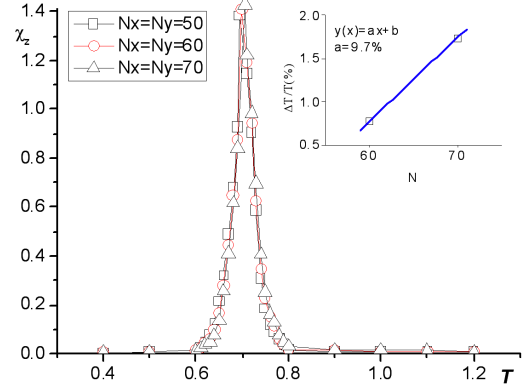

Fig. 3. The out-of-plane magnetic susceptibility vs. temperature for $J=1.00, K_{0}=1.00, \alpha=1, \beta=-0.05$, $D=0.115$ and three different lattice dimensions. The inset displays the relative variation of the critical temperature vs. lattice dimension.

\subsection{The influence of a non-uniform temperature dependence of anisotropy}

In this section we study the influence of a non-uniform temperature dependence of anisotropy on the magnetic phase transition (FM-PM) that characterizes the studied physical system. Thus, we propose a temperature dependence given by Eq. (3) and we assume three different values of the parameter $\beta=(-0.050 ;-0.055 ;-0.060)$, for a lattice characterized by the dimensions $N_{x}=N_{y}=50$, $N_{z}=1$. The other parameters that complete the properties of the studied system are $J=1.00, K_{0}=1.00$, $\alpha=1, D=0.115$. Consequently, in this case we take into account three different decreasing slopes respecting the linear temperature dependence of anisotropy (the linear character of this function is characterized by the coefficient $p=K_{0} \beta$ that determines the slope decreasing in Eq. (3)).

Figure 4 shows the out-of-plane magnetic susceptibility vs. temperature, where we observe a weak decrease of the critical temperature as a function of the slope $p$ (the temperature dependences of anisotropy taken into account, are presented in inset).

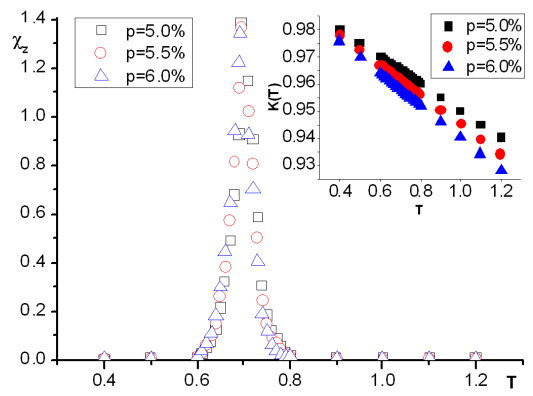

Fig. 4. The out-of-plane magnetic susceptibility vs. temperature for $J=1.00, K_{0}=1.00, \alpha=1, D=$ 0.115 and three different slopes decreasing $p$. The inset presents the anisotropy temperature dependences.

In Fig. 5 we present the specific heat behavior vs. temperature for the above mentioned physical conditions,

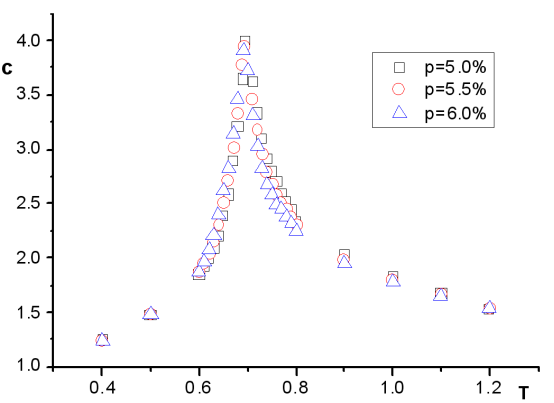

Fig. 5. The specific heat vs. temperature for $J=1.00$, $K_{0}=1.00, \alpha=1, D=0.115$ and three different slopes decreasing $p$.

TABLE

The relative variations of the critical temperature obtained from the study of susceptibility and specific heat, taking as reference the case $p=5 \%$.

\begin{tabular}{c|c|c}
\hline \hline$p[\%]$ & $(\Delta T / T)_{\chi}[\% \mathrm{o}]$ & $(\Delta T / T)_{\mathrm{c}}[\% \mathrm{o}]$ \\
\hline 5.5 & 2.016 & 1.872 \\
6.0 & 3.744 & 3.601
\end{tabular}

where we can observe a good agreement with the critical temperatures obtained from the study of susceptibility.

Therefore, in Table we calculate the comparative values of the critical temperature relative variation, taking as reference the case $p=5 \%$. Thus, the relative variations of the critical temperature obtained from the study of susceptibility and specific heat, respectively, are calculated with the expression

$$
(\Delta T / T)_{\chi, \mathrm{c}}=\frac{T_{\mathrm{cr}, 5 \%}-T_{\mathrm{cr}, p}}{T_{\mathrm{cr}, 5 \%}} .
$$

As a consequence of the above mentioned results, we can note that the decreasing temperature dependence of anisotropy implies the slight drop of the critical temperature, which means the magnetic ordered phase stability weakening. At the same time, this effect is enhanced by the increasing slope of the assumed linear variation of anisotropy parameter.

\section{Conclusions}

Using the Monte Carlo technique we studied a monolayer magnetic spins square lattice, characterized by a Hamiltonian that includes three different interactions (the direct exchange interaction, the crystalline anisotropy interaction and the long range dipolar interaction). We treated, in a comparative manner, the case of constant anisotropy interaction and the case of temperature dependent anisotropy, for different direct exchange parameter values. In this context we focused our numerical investigation on the magnetic properties of the above described system, taking into account the influence of 
the anisotropy characterized by a linear temperature dependency. Thus, our results reveal a slow decrease of the critical temperature that characterized the FM-PM magnetic phase transition, in comparison with the case of constant anisotropy. We can note that the temperature dependent anisotropy influence decreases with the direct exchange interaction parameter enhancement. On the other hand, we observe the magnetic ordered phase stability weakening along with the increasing slope of the anisotropy parameter variation as a function of temperature.

\section{References}

[1] Advanced Magnetic Nanostructures, Eds. D. Sellmyer, R. Skomski, Springer, New York 2006.

[2] Ultrathin Magnetic Structures, Eds. J.A.C. Bland, B. Heinrich, Vol. I, II, Springer, Berlin, 1994.

[3] Magnetic Anisotropies in Nanstructured Matter, Ed. P. Weinberger, CRC, Boca Raton 2008).

[4] M.T. Johnson, P.J.H. Bloemen, F.J.A. den Broeder, J.J. de Vries, Rep. Prog. Phys. 59, 1409 (1996).

[5] F.J. Himpsel, J.E. Ortega, G.J. Mankey, R.F. Willis, Adv. Phys. 47, 511 (1998).

[6] T. Sugimoto, T. Katayama, Y. Suzuki, M. Hashimoto, Y. Nishihara, A. Itoh, K. Kawanishi, J. Magn. Magn. Mater. 104, 1845 (1992).
[7] Z.S. Shan, J.X. Shen, R.D. Kirby, D.J. Sellmyer, Y.J. Wang, J. Appl. Phys. 75, 6418 (1994).

[8] M. Farle, W. Platow, A.N. Anisimov, B. Schultz, K. Baberschke, J. Magn. Magn. Mater. 165, 74 (1997).

[9] Y. Ashizawa, S. Saito, M. Takahashi, J. Appl. Phys. 91, 8240 (2002).

[10] C. Santamaria, H.T. Diep, J. Magn. Magn. Mater. 212, 23 (2000)

[11] D.P. Landau, K. Binder, A Guide to Monte Carlo Simulation in Statistical Physics, Cambridge University Press, Cambridge, 2000.

[12] M.E.J. Newman, G.T. Barkema, Monte Carlo Methods in Statistical Physics, Oxford University Press, Oxford 1999.

[13] N. Metropolis, A.W. Rosenbluth, M.N. Rosenbluth, A.M. Teller, E. Teller, J. Chem. Phys. 21, 1087 (1953).

[14] E. Birsan, R. Chis, Mod. Phys. Lett. B 24, 971 (2010).

[15] D.P. Pappas, K.-P. Kämper, H. Hopster, Phys. Rev. Lett. 64, 3179 (1990).

[16] H.Y. Zhang, Y.J. Wang, G.G. Zheng, J.X. Shen, Z.S. Shan, D.J. Sellmyer, IEEE Trans. Magn. 29, 3376 (1993). 